\title{
Genito-urinary rhabdomyosarcoma-challenges and controversies for the urologist
}

\author{
Eric Bortnick ${ }^{1}$, Jeffrey Stock $^{1,2}$, Fernando Ferrer ${ }^{1,2}$ \\ ${ }^{1}$ Department of Urology, Icahn School of Medicine at Mount Sinai, New York, USA; ${ }^{2}$ Kravis Children's Hospital at Mount Sinai, New York, NY, USA \\ Correspondence to: Fernando Ferrer, MD. Professor Urology, Icahn School of Medicine at Mount Sinai, New York, NY, USA. \\ Email: fernando.Ferrer@mountsinai.org.
}

Submitted Feb 04, 2020. Accepted for publication Jul 14, 2020.

doi: $10.21037 /$ tau-20-511

View this article at: http://dx.doi.org/10.21037/tau-20-511

Rhabdomyosarcoma (RMS) is the most common soft tissue sarcoma and originates from the genitourinary tract in $15-$ $20 \%$ of cases. Despite progress made by cooperative group studies in the United States and abroad, challenges and controversy remain in the management of these patients. In this article, we succinctly review some of the more significant questions facing urologists that participate in the care of children with RMS.

\section{Need to perform a retroperitoneal lymph node dissection (RPLND) for paratesticular tumors in boys greater than 10 years old-are we compliant with recommendations?}

Historically, all patients, regardless of age, underwent a RPLND if they were diagnosed with a paratesticular rhabdomyosarcoma (PTRMS) after an inguinal radical orchiectomy. To support the decision to perform an RPLND in all patients, Wiener et al. studied 121 patients from Intergroup Rhabdomyosarcoma Study (IRS) III with nonmetastatic PTRMS. The authors compared preoperative CT results for clinical nodal disease to pathological node results from the RPLND. Of the 121 patients, $81 \%$ were clinically node negative, however $14 \%$ of these patients had positive nodes found on RPLND pathology. Of the 19\% that were clinically node positive, $94 \%$ had positive nodes on pathology. Overall five-year survival was $91 \%$, with clinically N0 patients having a five-year survival of $96 \%$ compared to $69 \%$ of clinically $\mathrm{N} 1$ patients $(\mathrm{P}<0.001)(1)$.

While there was a benefit to RPLND shown, concerns about morbidity from the procedure led to a shift in thinking on management. Complications included intestinal obstruction (10\%), ejaculatory dysfunction (8\%), and lower extremity edema (5\%). As a result, in the IRSIV, patients that were clinically node negative on CT imaging did not undergo RPLND. In this review, patients with PTRMS had poorer outcomes if they were more than 10 years old (3-year failure free survival of $63 \% v s$. $90 \%$ ) (2).

Given the availability of these two subgroups, a comparison in outcomes was made between patients from IRSIIII and IRSIV by Wiener et al. Most notable was the difference in staging between the two cohorts, with $68 \%$ of IRSIII patients labeled as group 1 compared to $82 \%$ of IRS-IV patients, which is reflective of the reliability on CT in IRSIV patients, leading to understaging from decreased lymph node involvement recognition, compared to RPLND used in IRSIII patients. As a result, IRSIV led to "downgrouping" in $15 \%$ of patients from group 2 to group 1, leading to suboptimal treatment and a poorer 3 -year failure free survival on IRSIV, though this difference was not statistically significant (92\% in IRSIII compared to $86 \%$ on IRSIV, $\mathrm{P}=0.10$ ). When both IRSIII and IRSIV were combined, patients $>10$ years old had a poorer threeyear failure free survival compared with those $<10$ years old $(76 \%$ vs. $95 \%, \mathrm{P}<0.001)$, and poor 3 -year survival rate ( $99 \%$ for boys $<10$ years old $v s .82 \%$ in boys $>10$ years old $\mathrm{P}<0.001$ ) (3). As a result of these findings, management guidelines shifted to recommend RPLND in all boys $>10$ years old.

These findings were supported in further research studies. A SEER database study by Dang et al. of 255 patients with PTRMS from 1973-1999 found that the incidence of lymph node involvement was higher in older patients compared to younger ( $40 \%$ vs. $8 \%$, respectively). 
Furthermore, among 173 patients $>10$ years old, RPLND improved five-year overall survival rate from $64 \%$ to $86 \%$ $(\mathrm{P}<0.01)$ (4). In a review of 25 patients 10 years or older from the Malignant Mesenchymal Tumor Studies 84 and 98, Stewart et al. found that only one patient had a RPLND as initial therapy, and 16 patients had a relapse (14 of which were in the retroperitoneum), which could have been due to understaging. In this review, older age and primary tumor $>5 \mathrm{~cm}$ predisposed to a poorer outcome (5).

Even though the evidence exists, questions arose as to whether practice was following guideline recommendations. In a recent review by Hamilton $e t$ al., the SEER database for 111 adolescent patients with PTRMS from 1973-2012 was divided into groups of before the 2001 recommendations to perform RPLND in patients $>10$ years old and after. Five and ten-year overall survival for the entire cohort was $79 \%$ and $78 \%$ respectively. Incidence of $\mathrm{N} 1$ disease was $25 \%$. Those that underwent RPLND had a statistically significant improved five-year overall survival ( $92 \%$ vs. $64 \%, \mathrm{P}=0.003$ ), and RPLND was associated with an improved overall survival after adjusting for age, histology, stage at diagnosis, and race/ethnicity (HR 0.37, 95\% CI: 0.17-0.83). Despite this information and the guidelines, RPLND rates did not significantly increase after 2003 (45\% before 2003, 61\% after $2003, \mathrm{P}=0.09)$, with $39 \%$ of patients after 2003 not undergoing RPND (6).

\section{Is there a need to remove the scrotum if scrotal wall was violated during a paratesticular RMS surgery?}

The best practice for initial treatment of PTRMS is a radical orchiectomy via inguinal approach with resection of the spermatic cord to the level of the internal inguinal ring. However, circumstances exist where the initial treatment or biopsy of the tumor was done by a transscrotal approach, leading to fear of scrotal contamination, and debate remains on what to do in the secondary setting. Standard recommendations have included primary reexision by hemiscrotectomy with high resection of the spermatic cord. Some recent studies have questioned the need for this secondary procedure. In a retrospective review of 32 patients from the German Cooperative Soft Tissue Sarcoma Group, Italian Cooperative Group, and the Istituto Nazionale Tumori of Milan who underwent an initial incorrect scrotal approach, 24 patients underwent primary re-excision and eight did not. Of the 24 patients that underwent re-excision, residual tumor was found in only three. Of these 24 , twenty were alive in complete remission, two were alive and in remission after lung and lymph node recurrence, and two died of metastatic disease at the time of publication. All eight patients that did not undergo a re-excision were alive with no disease recurrence. Although promising data showing that it may not be necessary to perform a re-excision, the authors still emphasized the utility of hemiscrotectomy given the high risk of contamination with subsequent microscopic residual tumor (7).

In a review of 156 patients with embryonal PTRMS from Cooperative Soft Tissue Sarcoma Studies from 19862008, Seitz et al. found that $28 \mathrm{had}$ an initial transscrotal approach. Of those 28 patients, twelve were treated with hemiscrotectomy and sixteen without hemiscrotectomy. No difference was seen in five-year event-free survival (91.7\% vs. $93.8 \%$, respectively, $\mathrm{P}=0.662$ ). Relapse was seen in 3 of the patients that underwent hemiscrotectomy and in one that was treated without. Again, although the data seemed to suggest that hemiscrotectomy may not be necessary after transscrotal approaches, the authors stated that hemiscrotectomy should be performed if the scrotal skin was potentially contaminated (8).

A more recent single institution retrospective study by Hammond et al. seems to support the need for hemiscrotectomy. In a study of 49 patients referred to their center with PTRMS, 20 were initially treated with some form of scrotal violation. Of those twenty patients, fifteen received salvage therapy including some form of primary re-excision and radiation. $26.6 \%$ of patients treated with salvage therapy had disease recurrence $v s .80 \%$ of those patients not treated with salvage therapy ( $R R=3.0,95 \%$ CI: 1.16-7.73). However, when compared to patients that had appropriate initial therapy, there was no difference in disease-specific survival in patients that had an initial treatment with scrotal violation $(\mathrm{P}=0.86)$. Similarly, the progression-free survival was not statistically different between these two groups $(\mathrm{P}=0.68)$ (9). This question has to be considered on an individualized basis, reviewing the original procedure and therapeutic plans.

\section{Is there a need to operate on patients with mature rhabdomyoblasts?}

Initial treatment for pelvic rhabdomyosarcoma (PRMS) includes chemotherapy and radiation in an attempt to preserve GU organ function. Frequently after initial treatment, a residual mass can remain as complete response 
may not occur. It is common to find that the residual tumor has differentiated into a mature rhabdomyoblast, and questions remained about the need for radical excision of these tumors. The literature seems to point against excision. In a 2000 retrospective review of six patients with PRMS (4 bladder/prostate, 2 vulvovaginal) by Ortega et $a l$. in which initial follow up cystoscopy and biopsy after initial chemotherapy found presence of rhabdomyoblasts, all patients were alive without evidence of disease or further treatment 37-233 months after therapy ended. The number of rhabdomyoblasts appeared to decease in number on subsequent biopsies, and the authors stated that the presence of rhabdomyoblasts did not connote the presence of persistent malignant disease and did not demand further therapy (10). In a review of 72 patients with vaginal RMS from IRSI through IRSIV, the presence of rhabdomyoblasts after initial therapy signified chemotherapy response and further treatment consisted of continued chemotherapy instead of surgical re-excision (11). In a review of 44 patients from IRSIV with bladder/prostate RMS, Arndt et al. found that mature rhabdomyoblasts were not predictive of recurrence, and their presence did not justify radical surgery (12). In a summary of these findings by $\mathrm{Wu}$ et al., the authors stated that if the residual rhabdomyoblast can be resected without destroying a functional bladder, they can be removed, otherwise close follow-up with imaging is a reasonable alternative (13).

\section{What to do with a residual mass after treatment?}

A residual mass after chemo/radio therapy for RMS is a relatively common occurrence in group III patients, estimated to occur in about $20 \%$ of cases $(14,15)$. However, $50 \%$ of these are estimated to not harbor any viable tumor cells. Frequently, these masses consist of scar tissue and differentiated tumor cells (mature rhabdomyoblasts) that are believed to have little malignant potential (see above section on rhabdomyoblasts). Furthermore, Rodeberg et al. demonstrated that the presence of a residual mass post treatment did not impact EFS or OS in a group of patients from the COG studies. For those patients with recurrence, the pattern of recurrence was not related to the presence of a residual mass (14). As a result of this work we understand that radiographic response to therapy is not a predictor of outcome in RMS.

PET/CT is emerging as a useful tool in the management of RMS. A limited study from Memorial Sloan Kettering demonstrated that at 3-year median follow-up, local relapse free survival was improved among PET-negative vs. PETpositive patients $94 \%$ vs. $75 \%$. A follow-up publication by the same group showed that FDG PET could predict EFS, OS and local control $(16,17)$. The role of PET in evaluation of residual mass remains to be determined. A negative PET scan after therapy in a patient with a residual mass is reassuring. At this time, most see a scan interpreted as positive in this scenario as concerning and a recent review suggested that in these circumstances, case by case consideration must be given to what the appropriate next steps be may because of potential higher risk for recurrence.

Based on the above discussion points, surgical management of a residual mass is infrequently recommended, particularly when organ function would be sacrificed. Indications include pain, an enlarging mass, and organ dysfunction (an example of which would be persistent bladder outlet obstruction).

\section{Surgical outcomes related to bladder function- do we really know?}

Concerns about future bladder function remain after treatment of patients with PRMS. In 1994, Yeung et al. had eleven patients with PRMS undergo bladder function studies and upper tract evaluation at a mean of 6.6 years after treatment. All eleven patients received chemotherapy, and seven also were treated with radiation by either external beam or brachytherapy. All seven patients treated with radiation had reduced functional bladder capacity and abnormal voiding patterns including three with day and night incontinence and four with nocturnal enuresis. In contrast, all four patients that did not receive radiation had normal bladder capacity and voiding patterns (18). Using the gold-standard urodynamics, Soler et al. studied 8 patients with PRMS from a single institution from 1999-2003 that had been treated with multimodal therapy. Physical exam and urodynamics were performed at least six months after treatment (range, 6-39 months), and found that three asymptomatic patients had normal urodynamics, one had dysuria, and four had reduced bladder capacity of $33-52 \%$ resulting in overactivity with sensory urgency in three, and suprapubic pain during filling in one (19). In a more recent review by 20. Martelli et al., 22 patients from 1991-2007 with PRMS treated by partial cystectomy, partial prostatectomy, or both followed by brachytherapy were assessed for long term bladder function. A total of $13 / 22$ patients had a normal bladder function quality of life based on questionnaire, with nine reporting normal 
continence and four reporting rare dribbling. Urodynamics were performed on eleven of the patients, with the most common finding being detrusor sphincter dyssynergia (20).

Arndt et al. reviewed outcomes of 88 patients from IRSIV that underwent bladder preservation treatments for PRMS. For patients in this cohort, 55 had retained their bladder, and based on questionnaires completed by the patients, 36 had normal functioning bladders ( $40 \%$ of entire cohort). Abnormal function included dribbling/incontinence/ enuresis in ten, hydronephrosis requiring reimplantation in three, continent diversion or ureterostomy in three, and a urethral stricture in one (21). Given that the effects of radiation are delayed, it is possible that the number of normal functioning bladders may continue to decrease over time. It is clear that these patients require long term follow up.

\section{What is the best approach for post-treatment bladder dysfunction?}

Multimodal bladder preservation strategies utilizing chemotherapy, surgery, and radiation, each of which has the potential to damage bladder function, have undoubtedly resulted in preserved bladders with less than normal function. The diagnostic and medicinal approach to managing bladder dysfunction in this population includes familiar tools such as ultrasonography, urodynamics, postvoid residual and anticholinergic medications. Controversy surrounds appropriate long-term follow up regimens. The authors recommend baseline ultrasonography of the upper and lower tracts at the completion of therapy and annually during cancer follow up period, if asymptomatic. Patients with symptoms should undergo a more complete evaluation.

Patients who require cystectomy or cystoprostatectomy present another challenge related to timing of reconstruction. While performing reconstruction immediately after extirpative surgery is feasible, significant concerns regarding false negative margins at the time of reconstruction have led many to delay the procedure (22). In a recent review from the group in Padua, Italy, the authors express their preference for early reconstruction based on their experience suggesting that patients undergoing orthotopic neobladder that were reconstructed at the time of cystectomy obtained volitional voiding $(\mathrm{n}=4) v s$. those who underwent delayed reconstruction $(n=2)$. The authors speculate that scarring and greater difficulty of approximation of the proximal urethra to the reservoir may be responsible. The authors appropriately acknowledge that the limited numbers prevent definitive conclusions (23). Importantly, in their original publication on this subject the authors reported that 4/9 patients reconstructed experienced recurrence, including two with local recurrence despite negative margins on pathologic specimens (24). Regardless of the potential benefits related to continent reconstruction, the risk of local recurrence after extirpative surgery remains a concerning possibility.

\section{How to best attain local control?}

One of the ongoing challenges in the management of RMS is the issue of how to attain local control (25). This issue is of particular relevance for GU RMS for a few reasons: local relapse has been the most common type of failure in intermediate risk RMS; local failure portends a poor outcome; and in GU sarcoma the goal has been organ sparring. Surgery as a form of local control has the potential to drastically impact organ function. Additionally, previous publications have demonstrated the difficulty of accurately identifying negative margins intraoperatively in GU RMS, further complicating surgery as a definitive treatment for local control (26). With the understanding that radiation therapy is not without significant side effects, extirpative surgery which sacrifices whole organs has not been recommended. All of these factors have prompted recent studies by the Children's Oncology Group (COG) to focus on radiation therapy as a means to obtain local control, utilizing surgery as part of multimodal therapy when appropriate. The most recent studies from the COG, D903, ARST0531, as well as the current study ARST 1431 have varied in their dosing, method, and timing (early vs. late) of radiation, and all have used multiagent chemotherapy followed by radiation in attempting organ preservation.

The Intergroup Rhabdomyosarcoma Study Group (IRSG) and the COG have not been able to improve the outcome of Intermediate risk RMS patients (the majority of BP RMS) despite the addition of new chemotherapeutic agents and agent dose escalation (27). Three recent publications provide insight into our current outcomes utilizing the approach described above. Recently, COG ARST0531 studied if the addition of Irinotecan would improve outcomes over vincristine, dactinomycin, and cyclophosphamide (VAC) which has been standard treatment. Irinotecan is converted to its active metabolite SN-38 which functions a topoisomerase I inhibitor. Preclinical studies and a COG phase II (combination with vincristine) study reported significant activity. Hawkins 
et al., reporting for the COG, found that EFS was not improved by the addition of Irinotecan. No differential benefit was seen in patients when they were broken out between Embryonal or Alveolar RMS. Local control rates were not improved in this study, and radiation was begun at 4 weeks, unless patient age was less than 24 months (27).

A follow-up publication by Casey et al. focused specifically on the issue of local control in ARST0531 (25). The principle approaches to local control implemented in ARST0531 were the addition of Irinotecan, a potential radiosensitizer, and the early introduction of RT into the protocol at week 4 . Patients younger than 24 months were allowed to deviate from this protocol because of concerns regarding radiation-related morbidity. Irinotecan was given concomitantly with RT. Patients with embryonal or alveolar histology group III disease received a total of 50.4 Gy. Overall, a total of 369 patients with group III disease experienced a local failure rate of $27.9 \% v s$. $19.4 \%$ for embryonal RMS in D9803. Alveolar tumor local failure rate was 20.2 vs. 17.7. Local failure was not effected by histology, site, nodal status or proton $v s$. photon radiotherapy. Only tumor size $>5$ was a significant predictor of failure with rates of $16.7 \%$ for tumors $£ 5 v s .32 .3 \%$ for tumors $>5 \mathrm{~cm}$. The highest local failure rate was seen in 32 patients where radiation was omitted in which a local failure rate of $44.2 \%$ was observed. Disappointing as these results are, more frustrating is that the explanation for the increased local failure rates remains elusive. The authors considered multiple possibilities including the lower dose of cyclophosphamide in the ARST0531 protocol and variations in radiation administration, with no clear conclusions (25).

Proton beam therapy has been increasingly used in the treatment of RMS. The advantages of this mode of therapy include the ability to focus radiation at a specific depth, eliminating the pass-through effect and sparring normal tissues (28). This is particularly appealing when treating pelvic tumors such as BP RMS. Buszek et al. recently evaluated their experience with proton-based therapy for BP RMS, specifically looking at patterns of failure (28). They reported on 19 patients, the majority of which were in the intermediate risk group. Median tumor size was $3.2 \mathrm{~cm}$ $(37 \%>5 \mathrm{~cm})$. Proton beam therapy was given at a median of 15 weeks following chemotherapy. Median follow-up was 66.2 months. Four patients experienced local failure and all 4 subsequently died. The 5 -year local control rate was $76 \%$. The only significant risk factor for recurrence was tumor size $>5 \mathrm{~cm}$. These results are comparable with other series using photon based or intensity modulated (29-31). The study reports a favorable toxicity profile compared to other modalities suggesting that proton therapy may be equally efficacious with less toxicity.

In addition to changes in chemotherapy, the current COG study ARST1431 takes 2 different approaches to local control, delayed primary excision (DPE) and an increase in radiation for tumors $>$ than $5 \mathrm{~cm}$ that do not achieve complete response after chemotherapy or chemotherapy + DPE. DPE may be considered after 12 weeks of chemotherapy, and should be considered only if adequate margins without loss of form or organ function can be accomplished. If DPE is performed successfully the patient may qualify for a decreased dose of radiation according to the new protocol. In ARST1431 the total dose of radiation to patients with primary tumors $>5 \mathrm{~cm}$, that do not have a CR after chemotherapy +/- DPE will be increased to 59.4 Gy. Local treatment failures are often associated with poor survival thus local control remains a major challenge in our management of RMS.

\section{Risk group stratification-B/P RMS upstaging}

A frequent question regarding B/P RMS relates to staging. Specifically, why are B/P RMS almost always intermediate risk tumors? In part, the answer lies in our attempt to preserve organ function. Final risk group assignment for RMS is determined by a pretreatment TNM stage and surgical/pathological clinical group. The latter is assigned after the initial surgical procedure but before systemic therapy begins. The primary determinant of the clinical group is the presence of residual tumor. As a consequence of organ preservation strategies, almost all $\mathrm{B} / \mathrm{P}$ tumors undergo initial biopsy only, as extirpative procedures, which would likely result in organ dysfunction, are contraindicated. Thus, these tumors are then group III which places them in the intermediate risk group.

\section{How to obtain an appropriate Biopsy?}

The majority of patients undergoing treatment for GU RMS involving the bladder/prostate will fall into the intermediate risk group and not be candidates for upfront resection. In these patients, accurate diagnosis based on initial biopsy is the first step. While the majority of these lesions can be accessed endoscopically, adequate biopsy is not always easy. Artifact produced by cautery can make pathologic identification difficult. In such circumstances, it may be preferable to use the resectoscope to amputate 
part of the tumor and retrieve the specimen endoscopically, rather than taking a specimen the size of the resecting loupe from a pediatric scope. Additionally, in cases where this technique does not work, open intravesicle biopsy should be performed to confirm the diagnosis. If open biopsy is performed, concomitant lymph node sampling should be considered. In all instances and on-site pathologist should be available for consultation and to confirm that specimens are adequate for diagnosis before leaving the operating room.

COG intermediate risk protocols also entertain the possibility of transperineal percutaneous biopsy for B/P RMS. Several studies have suggested that the core needle biopsies can produce accurate results in the majority of pediatric neoplasms and provide adequate tissue for ancillary studies $(32,33)$. The authors experience would suggest that very careful planning with active involvement of institutional pathologists is required for this approach, lest inadequate tissue for diagnosis or ancillary studies be obtained. The theoretical risk of seeding a percutaneous biopsy tract is frequently raised, however more recent literature suggests that the risk is low (34). Additionally, the overwhelming majority of patients in current COG protocols go on to receive radiation at week 13 for local control.

\section{Do you manage infants with RMS the same as older children?}

Management of infants with RMS pose unique challenges because of their susceptibility to treatment morbidity. Radiotherapy in particular carries a high risk of offtarget effects in infants, limiting its utility as a local control modality. Given that the tumor biology does not appear to be different, it is thought that reduced survival demonstrated in some studies is related to a reduction in therapy $(35,36)$. These findings have prompted the new intermediate protocol to recommend that protocol dosing be adhered to in children less than 24 months of age (final decision is at the discretion of the local radiotherapist). The other alternative to be considered for these patients is surgical local control. This becomes a very individualized decision that should be made by the treatment team and family reviewing the risk and benefits of each approach.

\section{Conclusions}

When one considers that we began by using exenteration as the front-line approach, with poor survival, for children with RMS it is easier to appreciate the progress that has been made in the last few decades. This progress has been greatly facilitated by collaborative groups both in the US and abroad. The challenges discussed above, which are particularly germane to urologists, are some of the many focal points for improvement of our care for children with RMS.

\section{Acknowledgments}

Funding: None.

\section{Footnote}

Provenance and Peer Review: This article was commissioned by the Guest Editors (John Wiener, Jonathan Routh and Nicholas Cost) for the series "Pediatric Urologic Malignancies" published in Translational Andrology and Urology. The article was sent for external peer review organized by the Guest Editors and the editorial office.

Conflicts of Interest: All authors have completed the ICMJE uniform disclosure form (available at http://dx.doi. org/10.21037/tau-20-511). The series "Pediatric Urologic Malignancies" was commissioned by the editorial office without any funding or sponsorship. The authors have no other conflicts of interest to declare.

Ethical Statement: The authors are accountable for all aspects of the work in ensuring that questions related to the accuracy or integrity of any part of the work are appropriately investigated and resolved.

Open Access Statement: This is an Open Access article distributed in accordance with the Creative Commons Attribution-NonCommercial-NoDerivs 4.0 International License (CC BY-NC-ND 4.0), which permits the noncommercial replication and distribution of the article with the strict proviso that no changes or edits are made and the original work is properly cited (including links to both the formal publication through the relevant DOI and the license). See: https://creativecommons.org/licenses/by-nc-nd/4.0/.

\section{References}

1. Wiener ES, Lawrence W, Hays D, et al. Retroperitoneal node biopsy in paratesticular rhabdomyosarcoma. J Pediatr 
Surg 1994;29:171-7; discussion 178.

2. Crist WM, Anderson JR, Meza JL, et al. Intergroup rhabdomyosarcoma study-IV: results for patients with nonmetastatic disease. J Clin Oncol 2001;19:3091-2.

3. Wiener ES, Anderson JR, Ojimba JI, et al. Controversies in the management of paratesticular rhabdomyosarcoma: is staging retroperitoneal lymph node dissection necessary for adolescents with resected paratesticular rhabdomyosarcoma? Semin Pediatr Surg 2001;10:146-52.

4. Dang ND, Dang PT, Samuelian J, et al. Lymph node management in patients with paratesticular rhabdomyosarcoma: a population-based analysis. Cancer 2013;119:3228-33.

5. Stewart RJ, Martelli H, Oberlin O, et al. International Society of Pediatric Oncology: Treatment of children with nonmetastatic paratesticular rhabdomyosarcoma: results of the Malignant Mesenchymal Tumors Studies (MMT 84 and MMT 89) of the International Society of Pediatric Oncology. J Clin Oncol 2003;21:793-8.

6. Hamilton EC, Miller CC 3rd, Joseph M, et al. Retroperitoneal lymph node staging in paratesticular rhabdomyosarcoma-are we meeting expectations? J Surg Res 2018;224:44-9.

7. Dall'Igna P, Bisogno G, Ferrari A, et al. Primary transcrotal excision for paratesticular rhabdomyosarcoma: is hemiscrotectomy really mandatory? Cancer 2003;97:1981-4.

8. Seitz G, Dantonello TM, Kosztyla D, et al. Impact of hemiscrotectomy on outcome of patients with embryonal paratesticular rhabdomyosarcoma: results from the Cooperative Soft Tissue Sarcoma Group Studies CWS-86, 91, 96 and 2002P. J Urol 2014;192:902-7.

9. Hammond WJ, Farber BA, Price AP, et al. Paratesticular rhabdomyosarcoma: Importance of initial therapy. J Pediatr Surg 2017;52:304-8.

10. Ortega JA, Rowland J, Monforte H, et al. Presence of welldifferentiated rhabdomyoblasts at the end of therapy for pelvic rhabdomyosarcoma: implications for the outcome. J Pediatr Hematol Oncol 2000;22:106-11

11. Andrassy RJ, Wiener ES, Raney RB, et al. Progress in the surgical management of vaginal rhabdomyosarcoma: a 25-year review from the Intergroup Rhabdomyosarcoma Study Group. J Pediatr Surg 1999;34:731-4; discussion 734-5.

12. Arndt CA, Hammond S, Rodeberg D, et al. Significance of persistent mature rhabdomyoblasts in bladder/prostate rhabdomyosarcoma: Results from IRS IV. J Pediatr Hematol Oncol 2006;28:563-7.
13. Wu HY, Snyder HM 3rd, Womer RB. Genitourinary rhabdomyosarcoma: which treatment, how much, and when? J Pediatr Urol 2009;5:501-6.

14. Rodeberg DA, Stoner JA, Hayes-Jordan A, et al. Prognostic significance of tumor response at the end of therapy in group III rhabdomyosarcoma: a report from the children's oncology group. J Clin Oncol 2009;27:3705-11.

15. Borinstein SC, Steppan D, Hayashi M, et al. Consensus and controversies regarding the treatment of rhabdomyosarcoma. Pediatr Blood Cancer 2018. doi:10.1002/pbc.26809

16. Dharmarajan KV, Wexler LH, Gavane S, et al. Positron emission tomography (PET) evaluation after initial chemotherapy and radiation therapy predicts local control in rhabdomyosarcoma. Int J Radiat Oncol Biol Phys 2012;84:996-1002.

17. Casey DL, Wexler LH, Fox JJ, et al. Predicting outcome in patients with rhabdomyosarcoma: role of [(18)f] fluorodeoxyglucose positron emission tomography. Int J Radiat Oncol Biol Phys 2014;90:1136-42.

18. Yeung CK, Ward HC, Ransley PG, et al. Bladder and kidney function after cure of pelvic rhabdomyosarcoma in childhood, Br J Cancer 1994;70:1000-3.

19. Soler R, Macedo A Jr, Bruschini H, et al. Does the less aggressive multimodal approach of treating bladderprostate rhabdomyosarcoma preserve bladder function? J Urol 2005;174:2343-6.

20. Martelli H, Borrego P, Guérin F, et al. Quality of life and functional outcome of male patients with bladder-prostate rhabdomyosarcoma treated with conservative surgery and brachytherapy during childhood. Brachytherapy 2016;15:306-11.

21. Arndt C, Rodeberg D, Breitfeld PP, et al. Does bladder preservation (as a surgical principle) lead to retaining bladder function in bladder/prostate rhabdomyosarcoma? Results from intergroup rhabdomyosarcoma study iv. J Urol 2004;171:2396-403.

22. Ferrer F. Pediatric Urologic Oncology: Bladder and Testis. In: Partin AW, Dmochowski RR, Kavoussi LR, et al. editors. CAMPBELL-WALSH-WEIN UROLOGY, ed 12. Philadelphia, Elsevier, 2021:1111-27.

23. Castagnetti M, Herbst KW, Esposito C. Current Treatment of Pediatric Bladder and Prostate Rhabdomyosarcoma (Bladder Preserving vs Radical Cystectomy). Curr Opin Urol 2019;29:487-92.

24. Castagnetti M, Angelini L, Alaggio R, et al. Oncologic Outcome and Urinary Function After Radical Cystectomy for Rhabdomyosarcoma in Children: Role of the 
Orthotopic Ileal Neobladder Based on 15-year Experience at a Single Center. J Urol 2014;191:1850-5.

25. Casey DL, Chi YY, Donaldson SS, et al. Increased local failure for patients with intermediate-risk rhabdomyosarcoma on ARST0531: A report from the Children's Oncology Group. Cancer 2019;125:3242-8.

26. Merguerian PA, Agarwal S, Greenberg M, et al. Outcome analysis of rhabdomyosarcoma of the lower urinary tract. J Urol 1998;160:1191-4; discussion 1216.

27. Hawkins DS, Chi YY, Anderson JR, et al. Addition of Vincristine and Irinotecan to Vincristine, Dactinomycin, and Cyclophosphamide Does Not Improve Outcome for Intermediate-Risk Rhabdomyosarcoma: A Report From the Children's Oncology Group. J Clin Oncol 2018;36:2770-7.

28. Buszek SM, Ludmir EB, Grosshans DR, et al. Patterns of failure and toxicity profile following proton beam therapy for pediatric bladder and prostate rhabdomyosarcoma. Pediatr Blood Cancer 2019;66:e27952.

29. Vern-Gross TZ, Indelicato DJ, Bradley JA, et al. Patterns of failure in pediatric rhabdomyosarcoma after proton therapy. Int J Radiat Oncol Biol Phys 2016;96:1070-7.

30. Childs SK, Kozak KR, Friedmann AM, et al. Proton radiotherapy for parameningeal rhabdomyosarcoma:

Cite this article as: Bortnick E, Stock J, Ferrer F. Genitourinary rhabdomyosarcoma-challenges and controversies for the urologist. Transl Androl Urol 2020;9(5):2422-2429. doi:10.21037/tau-20-511 clinical outcomes and late effects. Int J Radiat Oncol Biol Phys 2012;82:635-42.

31. Curtis AE, Okcu MF, Chintagumpala M, et al. Local control after intensity-modulated radiotherapy for headand-neck rhabdomyosarcoma. Int J Radiat Oncol Biol Phys 2009;73:173-7.

32. Garrett KM, Fuller CE, Santana VM, et al. Percutaneous Biopsy of Pediatric Solid Tumors. Cancer 2005;104:644-52.

33. Chowdhury T, Barnacle A, Haque S et al. UltrasoundGuided Core Needle Biopsy for the Diagnosis of Rhabdomyosarcoma in Childhood. Pediatr Blood Cancer 2009;53:356-60.

34. Berger-Richardson D, Swallow CJ. Needle Tract Seeding After Percutaneous Biopsy of Sarcoma: Risk/benefit Considerations. Cancer 2017;123:560-7.

35. Ferrari A, Casanova M, Bisogno G, et al. Italian Cooperative Group: Rhabdomyosarcoma in infants younger than one year old: a report from the Italian Cooperative Group. Cancer 2003;97:2597-604.

36. Malempati S, Rodeberg DA, Donaldson SS, et al. Rhabdomyosarcoma in infants younger than 1 year: a report from the Children's Oncology Group. Cancer 2011;117:3493-501. 